\title{
Estimating Geriatric Mortality after Injury Using Age, Injury Severity, and Performance of a Transfusion: The Geriatric Trauma Outcome Score
}

\author{
Frank Z. Zhao, MD, Steven E. Wolf, MD, ${ }^{2}$ Paul A. Nakonezny, PhD, ${ }^{3}$ Abu Minhajuddin, PhD, ${ }^{3}$ \\ Ramona L. Rhodes, MD, MPH, ${ }^{4}$ M. Elizabeth Paulk, MD, and Herb A. Phelan, MD, MSCS ${ }^{2}$
}

\begin{abstract}
Background: A tool to determine the probability of mortality for severely injured geriatric patients is needed. Objective: We sought to create an easily calculated geriatric trauma prognostic score based on parameters available at the bedside to aid in mortality probability determination.

Methods: All patients $\geq 65$ years of age were identified from our Level I trauma center's registry between January 1, 2000 and December 31, 2013. Measurements included age, Injury Severity score (ISS), units of packed red blood cells (PRBCs) transfused in the first 24 hours, and patients' mortality status at the end of their index hospitalization. As a first step, a logistic regression model with maximum likelihood estimation and robust standard errors was used to estimate the odds of mortality from age, ISS, and PRBCs after dichotomizing PRBCs as yes/no. We then constructed a Geriatric Trauma Outcome (GTO) score that became the sole predictor in the re-specified logistic regression model.

Results: The sample $(\mathrm{n}=3841)$ mean age was $76.5 \pm 8.1$ years and the mean ISS was $12.4 \pm 9.8$. In-hospital mortality was $10.8 \%$, and $11.9 \%$ received a transfusion by 24 hours. Based on the logistic regression model, the equation with the highest discriminatory ability to estimate probability of mortality was GTO Score $=$ age + $(2.5 \times$ ISS $)+22$ (if given PRBCs). The area under the receiver operating characteristic curve (AUC) for this model was 0.82 . Selected GTO scores and their related probability of dying were: $205=75 \%, 233=90 \%$, $252=95 \%, 310=99 \%$. The range of GTO scores was 67.5 (survivor) to 275.1 (died).

Conclusion: The GTO model accurately estimates the probability of dying, and can be calculated at bedside by those possessing a working knowledge of ISS calculation.
\end{abstract}

\section{Introduction}

A PPROXIMATELY 500,000 GERIATRIC PATIENTS are admitted to trauma centers after injury on an annual basis. ${ }^{1,2}$ Due to decreased physiological reserve, frailty, and preinjury comorbidities these patients have higher morbidity and mortality on an injury-for-injury basis than their younger counterparts. $^{3-6}$ Advances in pre-hospital transport and resuscitation over the last 20 years have resulted in progressively sicker trauma patients arriving to the intensive care unit (ICU). Although this is gratifying, these more severely injured patients go on to consume tremendous amounts of resources and their care frequently entails undergoing aggressive, invasive interventions. Despite all of these efforts, many severely injured geriatric patients suffer through a prolonged ICU course only to subsequently die.

It is heartening that the historical resistance of surgeons to involving palliative care in the management of their surgical patients has been crumbling in recent years. ${ }^{7-9}$ The culture of surgical training in years past frequently glorified heroic attempts at rescue, exalting those who would "go down swinging" while dismissing talk of palliative care as "giving

\footnotetext{
${ }^{1}$ Department of General Surgery; ${ }^{2}$ Division of Burns/Trauma/Critical Care, Department of Surgery; ${ }^{4}$ Division of Geriatrics and Palliative Care, Department of Internal Medicine; ${ }^{5}$ Division of Palliative Care, Department of Internal Medicine, UT Southwestern Medical Center, Parkland Memorial Hospital, Dallas, Texas.

${ }^{3}$ Division of Biostatistics, Department of Clinical Sciences, UT Southwestern Medical Center, Dallas, Texas.

Accepted April 27, 2015.

(c) Frank Z. Zhao, Steven E. Wolf, Paul A. Nakonezny, Abu Minhajuddin, Ramona L. Rhodes, M. Elizabeth Paulk, Herb A. Phelan 2015; Published by Mary Ann Liebert, Inc. This Open Access article is distributed under the terms of the Creative Commons Attribution Noncommercial License (http://creativecommons.org/licenses/by-nc/4.0/) which permits any noncommercial use, distribution, and reproduction in any medium, provided the original author(s) and the source are credited.
} 
up." The shift away from this crude view to a more enlightened one is best exemplified by the Center to Advance Palliative Care's "Improving Palliative Care in the ICU" (IPAL-ICU) project. Our trauma surgeons are full-throated advocates for this approach to our patient population both overall as well as for geriatric trauma patients in particular.

As we have attempted to bring the principles of palliative care to the bedside for our severely injured geriatric trauma patients, we have struggled with the fact that quantitative prognostic tools to aid proxies in their decision making about goals of care do not exist. As a result, prognostic information is usually delivered based on the provider's subjective experience rather than being driven by evidence. Our group has previously attempted to create a quantitative scoring system for geriatric trauma patients' mortality rates for the index admission based on age, Injury Severity score (ISS), and the presence of comorbidities utilizing a large national database. $^{10}$ This attempt was unsuccessful, however, in part due to a lack of consideration for any markers of physiological distress. The present investigation is the next in our series as we try to develop a Geriatric Trauma Outcome (GTO) score that will be simple to understand, easy to calculate, and highly accurate.

\section{Methods}

\section{Study settings and measures}

After institutional review board (IRB) approval, our local trauma registry was queried for all trauma admission patients to our urban, Level I trauma center between January 1, 2000 and December 31, 2013 who were age 65 years or older. Data fields captured were each subject's age in years, ISS, amount of packed red blood cells (PRBCs) transfused during their first 24 hours after admission for traumatic injury, and their mortality status at the end of their index hospitalization.

Since its inception in the $1970 \mathrm{~s},{ }^{11}$ the ISS has been the gold standard language used by all trauma providers to communicate severity of injury. The ISS divides the body into six regions (head and neck, face, chest, abdomen, extremities, and external), and each region is assigned an Abbreviate Injury score, or AIS, of 0 (uninjured) to 5 (maximally injured while still being compatible with life) based on a priori definitions. The three worst AISs are squared and the sum of these three squares constitutes the ISS for that patient. An uninjured person has an ISS of 0 , whereas the maximum ISS that a patient can have is 75 (i.e., $5^{2}+5^{2}+5^{2}$ ).

\section{Statistical analysis}

As a first step, a logistic regression model with maximum likelihood estimation and robust standard errors (Huber Sandwich Estimator) was used to estimate the odds of mortality from age, ISS, and whether PRBCs were transfused in the first 24 hours after injury after dichotomization of PRBCs as a binary indicator (i.e., yes or no for whether any amount of transfusion occurred). As a second step, and on the basis of the initial analysis along with the estimated coefficients for age, ISS, and PRBCs, we constructed a GTO score that became the sole predictor in the re-specified logistic regression model to estimate the probability of mortality. Finally, for the re-specified logistic model, the area under the receiver operating characteristic curve (AUC) was estimated to evaluate how well the GTO score discriminated mortality status. The AUC for the GTO score was tested against a nominal area of 0.50 using the $\mathrm{Z}$ statistic.

All statistical analyses were carried out using SAS software, version 9.4 (SAS Institute, Inc., Cary, NC). The level of significance was set at $\alpha=0.05$ (two-tailed).

\section{Results}

\section{Patient characteristics}

The sample consisted of 3841 subjects, with a mean age of 76.5 years (standard deviation $[\mathrm{SD}] \pm 8.1$ ) and mean ISS of $12.4(\mathrm{SD} \pm 9.8)$. Overall in-hospital mortality was $10.8 \%$, and $11.9 \%$ received a PRBC transfusion within the first 24 hours of hospitalization.

\section{Logistic regression}

The results of fitting the initial logistic regression model containing age, ISS, and PRBCs (yes/no) are shown in Table 1A. Based on this initial logistic regression model, and as shown in Table 1A, the estimated coefficients for ISS and PRBCs were expressed relative to age so as to specify the following equation to estimate the probability of mortality:

$$
\text { GTO score }=\text { Age }+(2.5 \times \text { ISS })+22 \text { (if given PRBCs })
$$

[Equation 1]

which we refer to here as the GTO score.

As a second step, we then used the GTO score as the sole predictor in a re-specified logistic regression model to estimate the probability of mortality. The results of fitting this respecified GTO logistic regression model can be found in Table 1B. The fitted GTO logistic model is shown below:

$$
\text { GTO logistic model }=\frac{e^{-6.9115+(0.03912 x G T O)}}{1+e^{-6.9115+(0.03912 x G T O)}}
$$

[Equation 2]

Table 1. Results of the Initial and Re-Specified Logistic Regression MODELS FOR THE Geriatric Trauma Outcome Score

A

Initial model

\begin{tabular}{lrcc}
\hline Effect & Estimate & Standard error & $\mathrm{P}$ \\
\hline Intercept & -6.0664 & 0.6022 & $<0.0001$ \\
Age & 0.03949 & 0.007101 & $<0.0001$ \\
ISS & 0.09749 & 0.005920 & $<0.0001$ \\
PRBCs (1 versus 0) & 0.87120 & 0.1443 & $<0.0001$ \\
\hline
\end{tabular}

$B$

Re-specified model

\begin{tabular}{lccc}
\hline Effect & Estimate & Standard error & $\mathrm{P}$ \\
\hline Intercept & -6.91150 & 0.250400 & $<0.0001$ \\
GTO score & 0.03912 & 0.001888 & $<0.0001$ \\
\hline
\end{tabular}

GTO, Geriatric Trauma Outcome score; ISS, Injury Severity score; PRBCs, packed red blood cells. 


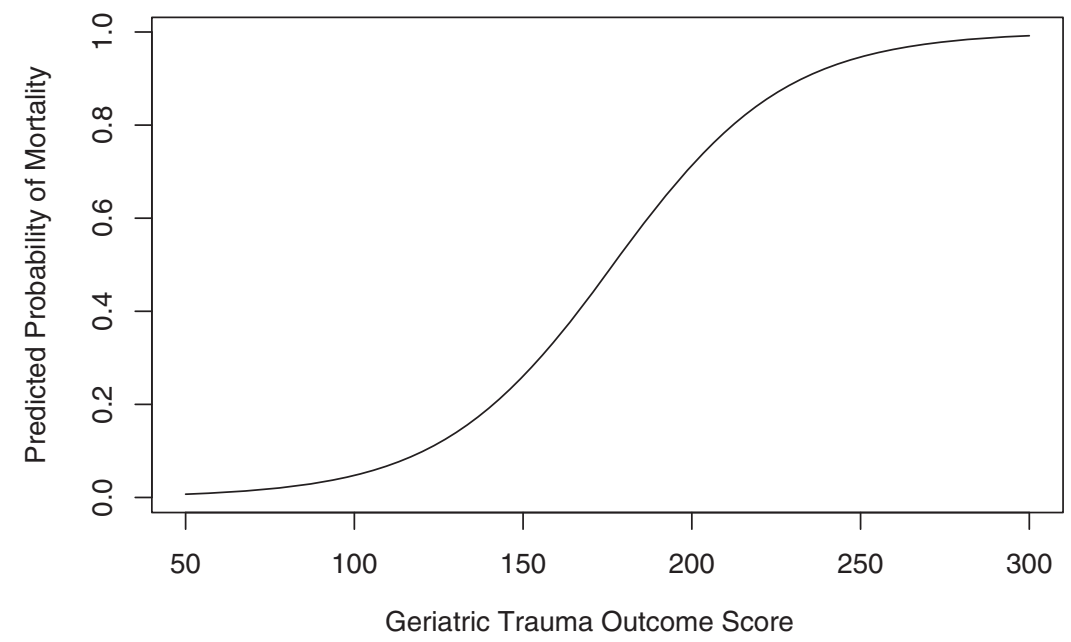

FIG. 1. The sigmoid-shaped curve showing the predicted probability of mortality across the spectrum of Geriatric Trauma Outcome scores.

The AUC for this re-specified GTO logistic model was 0.82 $(p<0.0001)$. The misclassification (error) rate for the GTO logistic model was $9.79 \%$, and the Brier score was 0.07 .

Selected GTO scores and their related probability of dying during the index admission were estimated as (GTO score $=$ probability of dying): $70=1.5 \%, 177=50 \%, 205=75 \%$, $233=90 \%, 252=95 \%, 310=99 \%$. The range of observed GTO scores in the sample was 67.5 (survivor) to 275.1 (died). The lowest and highest observed GTO scores and related probability of dying for any survivor was 67.5 (1.3\%) and $270.2(97.5 \%)$, respectively. Likewise, the lowest and highest observed GTO score and related probability of dying for any patient who died was 75.5 (1.8\%) and 275.1 (97.9\%) (Fig. 1).

\section{Discussion}

The GTO score shows high discriminative ability to predict inpatient mortality for elderly patients after admission for injury using the patient's age, ISS, and performance of a blood transfusion as variables. As an example calculation, take the hypothetical case of a 78-year-old patient with a large frontal contusion and craniotomy $(\mathrm{AIS}=5)$, unilateral flail chest with multiple rib fractures (AIS $=4$ ), and a midshaft femur fracture (AIS = 3) who received two units of blood during his/her resuscitation. These injuries result in an ISS of $5^{2}+4^{2}=3^{2}=50$. The GTO score would therefore be $78+[50 \times 2.5]+22=225$, which corresponds to a predicted probability of mortality of $87 \%$ for the index admission (Fig. 1). This score can be quickly calculated at the bedside at the time of initial ICU arrival by anyone facile with the calculation of ISSs (as all trauma surgeons are). If a nonsurgeon is providing assistance with counseling or the delivery of palliative care services, the ISS can be easily communicated making the multidisciplinary calculation of the GTO score an easy matter. It therefore has potential utility as a decision aid for any provider counseling proxy decision makers about treatment choices after severe levels of geriatric injury. We have created a convenient nomogram to assist in the calculations (Fig. 2).

Caring for geriatric trauma patients frequently involves medically and ethically complex decision making as goals of care often vary in this population. Choices must often be made between aggressive regimens with invasive interventions to pain and symptom control with or without provision of end-oflife care. Before making these enormous decisions (with frequently irreversible consequences), geriatric trauma patients or their proxy decision makers universally ask for prognostic information. Presently, limited work has been done in the field of prognostic tools after geriatric trauma and the patient/proxy must rely instead on the subjective impressions of the clinician.

One of the goals of a score that predicts odds of mortality across a spectrum of injury severity is that it can potentially assist in determinations of futility of care. Providers seeking medical guidance about post-trauma futility determinations have a limited body of work from which to draw. The National Association of EMS Physicians published guidelines in 2012 for pre-hospital providers on the withholding of attempts at resuscitation (briefly, apnea, pulselessness, and no organized electrical activity on EMS arrival). ${ }^{12}$ Additionally, the burn community has utilized a scoring system since the 1960s for futility of care after thermal injury in which the sum of a

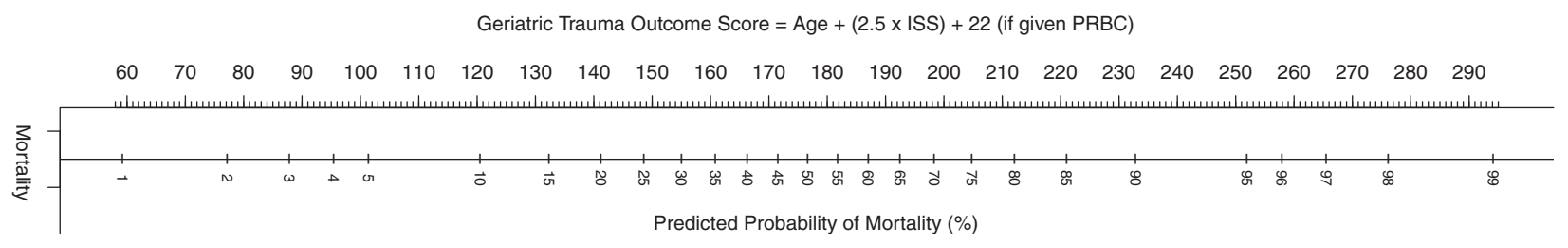

FIG. 2. Clinically useful nomogram for the predicted probability of mortality across the spectrum of Geriatric Trauma Outcomes scores. 
patient's age and percent total body surface area burned (with the recent addition of 17 points for an inhalation injury ${ }^{13}$ ) indicate futility when a score of 130 is reached. ${ }^{14}$ For the general trauma population that arrives to the hospital, however, a well-accepted definition of futility has been elusive.

Part of this elusiveness is due to the notion of futility itself as it means different things to different providers as well as the general public. Universal lethality for a given injury is actually rarer than one would expect short of intuitive injuries such as decapitation or hemicorpectomy. Experienced trauma surgeons all have "battle stories" about massively injured patients whom they expected to die but pulled through. Once the chest-thumping is finished, however, we are left to ask ourselves how this anecdote should inform our future practice and where the line should be redrawn if at all. This is especially problematic given that failed trauma resuscitation often does not just mean the patient's death, but very often leaving the patient in a persistent vegetative state with a remaining lifespan to be spent bed-bound at a skilled nursing facility. Currently, these experiences are usually what guide trauma providers when counseling families after injury. The lay public comes to these discussions from a viewpoint that was investigated by Jacobs and colleagues through random digit dialing of 1006 respondents across the Unites States. In responses that were weighted to match U.S. Census demographic data, ${ }^{15}$ they found that $50 \%$ of respondents would want a person pronounced dead at the scene of injury to be transported to a trauma center, with $47 \%$ citing the hope that something could be done as the reason. For patients admitted to the hospital, a majority of the public $(72 \%)$ felt that lifesustaining treatment should be stopped when "doctors believe there is no hope for recovery," and had a relatively high level of trust in a physician's ability to make this determination (mean of 7.0 on a scale of 1 to 10 where 1 was no trust at all and 10 was trust completely).

This difficulty in defining futility is one of the main strengths of the GTO score. Because it reports odds of death as a continuous outcome, it does not presume to define futility for the user. It simply reports the likelihood of survival with a high degree of accuracy and the user is left to decide if, say, a $93 \%$ probability of death constitutes futility. It offers the additional value of being easy to calculate, and the score should be available relatively early in a patient's hospital course. Additionally, the variables are not subject to missing data for the caregiver standing at the bedside, meaning that it is universally applicable. It is our hope that the reporting of a point estimate of death will be better accepted by proxy decision makers who can be otherwise frustrated when caregivers cite literature about certain findings that suggest an "increased likelihood" of a given poor outcome without being quantitative about the magnitude of the effect.

Our study has three primary limitations. Geriatric medicine has recognized for some time that the notion of frailty is more important than simple age in evaluating and treating elderly patients. The surgical world has been slower to make this connection, however, and the GTO score does not take this into account. Our group recently demonstrated that retrospective comorbidity data from the American College of Surgeons National Trauma Databank was inadequate to serve as a source in investigating this aspect of geriatric trauma. ${ }^{10}$ Recent work has been done by Joseph and colleagues who have attempted to assess the impact of frailty on geriatric outcomes after trauma. Using a validated 15-item survey composed exclusively of questions related to preinjury comorbidities and function, they have found that frailty as defined by their tool is associated with increased likelihood of death, complications, and discharge to skilled nursing for the index admission after geriatric trauma. ${ }^{16-18}$ Known as the Trauma-Specific Frailty Index, it has the key feature of addressing directly the issue of preinjury frailty. Due to its requirement of a knowledgeable historian, and questions regarding the sexual function and emotional state of the patient, it has the potential for bias when a proxy is the data source. It also does not take into account the anatomy and magnitude of injury, which is obviously key when making prognostic statements. Finally, it serves as a risk stratifier, therefore giving qualitative rather than quantitative input. Despite these limitations, however, it serves not only to help in counseling proxy decision makers but it has also serves as a clarion call for trauma surgeons to begin to shift our thinking away from age and toward the importance of frailty when caring for the elderly trauma patient.

Additionally, we selected the three variables of age, ISS, and transfusion a priori. These variables were chosen due to their ease of identification and the fact that they are universally known by the time of admission to the ICU. It is an unfortunate fact that other data related to vital signs, lab tests, or historical information are frequently missing not at random (MNAR) in the most critically ill of trauma patients, and therefore any model reliant on these data points would be markedly limited.

The final limitation of the GTO score is that it only deals with mortality at the time of the index admission. When counseling proxies after severe geriatric trauma, they usually desire information not only about the likelihood of survival, but expected function and quality of life as well. Although helpful for its ability to prognosticate in the short term, the GTO score lacks data regarding long-term outcomes in those who survive to discharge. This information is arguably more important to patients or their proxies when making treatment goal decisions than survival of the index admission. As the retrospective data cannot be merged with Medicare claims data, long-term outcomes on a score-for-score basis remain unknown.

Our future work is designed to address both of these weaknesses. Our group is currently in the early stages of establishing a collaborative effort with Dr. Joseph's team with the intent of prospectively investigating long-term outcomes after geriatric trauma so that those findings may inform our models. Additionally, we intend to ascertain whether a hybrid model incorporating the anatomical and physiological aspects of the GTO score with the comorbidity and function aspects of the Trauma-Specific Frailty Index may result in a new, synergistic model with heightened discriminatory abilities for predicting geriatric outcomes after trauma.

In summary, the Geriatric Trauma Outcome Score accurately predicts continuous odds of mortality across a spectrum of severities of injury. It has potential as a decision aid when counseling proxy decision makers on treatment choices after injury in the elderly.

\section{Acknowledgments}

We would like to thank Cari Stebbins for her assistance with the administrative aspects of obtaining and maintaining approval from the UT Southwestern IRB for this study. 
This work has been accepted as a poster at the May, 2015 meeting of the American Geriatrics Society in National Harbor, MD.

\section{Author Disclosure Statement}

No competing financial interests exist.

\section{References}

1. Thompson HJ, McCormick SH, Kagan SH: Traumatic brain injury in older adults: epidemiology, outcomes, and future implications. J Am GeriatrSoc 2006;54:1590-1595.

2. Rice DP, MacKenzie EJ, Jones AS, et al.: Cost of Injury in the United States: A Report to Congress. San Francisco: Institute for Health and Aging, University of California, and Injury Prevention Center, Johns Hopkins University, 1989.

3. Hukkelhoven CW, Steyerberg EW, Rampen AJ, et al.: Patient age and outcome following severe traumatic brain injury: an analysis of 5,600 patients. J Neurosurg 2003; 99:666-673.

4. Bergeron E, Lavoie A, Clas D, et al.: Elderly trauma patients with rib fractures are at greater risk of death and pneumonia. J Trauma 2003;54:478-485.

5. Demetriades D, Sava J, Alo K, et al.: Old age as a criterion for trauma team activation. J Trauma 2001;51:754-756.

6. Joseph B, Zangbar B, Pandit V, et al.: Mortality after trauma laparotomy in geriatric patients. J Surg Res 2014;190: 662-666.

7. Bradley CT, Weaver J, Brasel KJ: Addressing access to palliative care services in the surgical intensive care unit. Surgery 2010;147:871-877.

8. Schwarze ML, Bradley CT, Brasel KJ: Surgical "buy-in": the contractual relationship between surgeons and patients that influences decisions regarding life-supporting therapy. Crit Care Med 2010;38:843-848.

9. Wood GJ, Arnold RM: How can we be helpful? Triggers for palliative care consultation in the surgical intensive care unit. Crit Care Med 2009;37:1147-1148.
10. Duvall DB, Zhu X, Elliott AC, et al.: Injury severity and comorbidities alone do not predict futility of care after geriatric trauma. J Palliat Med 2015; 18:246-250.

11. Baker SP, O'Neill B, Haddon Jr W, et al.: The Injury Severity Score: a method for describing patients with multiple injuries and evaluating emergency care. J Trauma 1974;14:187-196.

12. National Association of EMS Physicians Position Statement: Withholding of Resuscitation for Adult Traumatic Cardiopulmonary Arrest. PrehospEmerg Care 2013;17:291.

13. Roberts G, Lloyd M, Parker M, et al.: The Baux score is dead. Long live the Baux score: a 27-year retrospective cohort study of mortality at a regional burns service. J Trauma Acute Care Surg 2012;72:251-256.

14. Baux S: Contribution al'etude du traitement local des bruluresthermiquesetendues, These, Paris, 1961.

15. Jacobs LM, Burns K, Jacobs BB: Trauma death: views of the public and trauma professionals on death and dying from injuries. Arch Surg 2008;143:730-735.

16. Joseph B, Pandit V, Zangbar B, et al.: Superiority of frailty over age in predicting outcomes among geriatric trauma patients: a prospective analysis. JAMA Surgery 2014;149: 766-772.

17. Joseph B, Pandit V, Zangbar B, et al.: Validating traumaspecific frailty index for geriatric trauma patients: a prospective analysis. J Am Coll Surg 2014;219:10-17.

18. Joseph B, Pandit V, Rhee P, et al.: Predicting hospital discharge disposition in geriatric trauma patients: is frailty the answer? J Trauma Acute Care Surg 2014;76:196-200.

Address correspondence to: Herb A. Phelan, MD, MSCS

University of Texas-Southwestern Medical Center

Parkland Memorial Hospital Division of Burns/Trauma/Critical Care 5323 Harry Hines Boulevard, E5.508A Dallas, TX 75390-9158

E-mail: herb.phelan@utsouthwestern.edu 